\title{
Efficient Power-Aware Network Provisioning for All-Optical Multicasting in WDM Mesh Networks
}

\author{
Ashraf M. Hamad \\ Microsoft Corporation \\ One Microsoft Way, Redmond, WA 98052-6399, USA \\ Email: ahamad@microsoft.com
}

\author{
Ahmed E. Kamal \\ Department of Electrical and Computer Engineering \\ Iowa State University, Ames, IA 50011-3060, USA \\ Email: kamal@iastate.edu
}

\begin{abstract}
Optimal network provisioning is the process of equipping the network with the devices and resources needed to support all traffic demands while minimizing the network cost. Optical Amplifiers (OAs) are one of those devices that contribute significantly to the network cost, especially in wide area networks. We propose a heuristic approach for placing the minimum number of OAs in the network. The solution is designed for All-Optical Multicasting and is based on dividing the problem into smaller subproblems. While solving them separately, the heuristic takes the interdependency between these subproblems into consideration. Therefore, efficient and accurate solutions can be obtained which is shown by the numerical results.
\end{abstract}

\section{INTRODUCTION}

Supporting one-to-many communication using All Optical Multicasting (AOM) [1] in wavelength-routing optical networks relies on the use of light-tree [2] and light-forest [3] concepts. In a nutshell, AOM aims to achieve signal transparency by keeping the signal in the optical domain while in-transit within the network. Branching at the intermediate nodes of the light-trees and light-forests is achieved by using passive optical splitters which can be configured to split the power of the incoming signal into two or more outgoing links.

Due to this splitting loss, AOM directly impacts the system power budget, which leads to the need for more OAs to compensate for the power loss. This impact was addressed in the literature as the Optical Amplifiers Placement (OAP) problem. The OAP is a network provisioning problem that aims to place the minimum number of OAs in the network while satisfying the connectivity requirements of all the sessions. It was addressed at different network topologies, including the point-to-point [4] and ring networks [5]. However, it is more involved in wavelength-routing mesh networks as it includes three integrated subproblems, i.e., Routing (R), Wavelength Assignment (WA) and Power Assignment (PA) subproblems.

The authors proposed a Mixed Integer Linear Program (MILP) in [6] that solves the R, WA and PA subproblems in an integrated way for the AOM traffic while the network cost is represented as the total power amplification needed in the network. For the unicast traffic, the OAP problem in the simpler broadcast-and-select architecture was addressed in [7]. The problem was solved using Mixed Integer non-Linear

This research was supported in part by grants CNS-0626741 and ANI0087746 from the National Science Foundation.
Programming (MInLP) with the objective of minimizing the total number of OAs. The problem in [7] was generalized in [8] by incorporating different layout topologies (stars, trees and/or rings) and by considering the fact that the OAs' cost is location dependent. The solution is based on the simulation annealing technique which was also employed in [9] to jointly solve the RWA and OAs placement problems in power-grids based WDM networks. While the studies in [6]-[9] considered the case of unequally powered-signals at the entry point of the OAs, reference [10] solved the equal powered-signals instance of the problem proposed in [7] using MILP.

We focus in this paper on the OAP problem for supporting AOM in the backbone optical networks where the network cost is dominated by the OAs cost. Therefore the goal of our study is to minimize the number of OAs. We propose an efficient heuristic that provides near optimal solutions by using a set of routing cost functions that are defined in terms of the OAs number. These functions are used to determine the routing decisions of the sessions based on the required number of OAs which reflects positively on the solutions quality.

The rest of the paper is organized as follows. The system model is first introduced in the next section. We then present the heuristic details and numerical results in Sections III and IV, respectively. Finally, the paper is concluded in Section V.

\section{System Model AND Assumptions}

Two system power constraints are considered in our study. First, the power strength of each channel is not allowed to fall below a specific sensitivity level, called $P_{S e n}$. Second, the aggregate power strengths of all the wavelengths should always be below a specific upper-bound, called $P_{M A X}$.

The power strengths of all the channels used over a specific link are assumed to be equal. Power symmetry is achieved by equipping each output port of all the optical cross connects (OXCs) with an equalizer. This assumption is used to help reducing the number of OAs by avoiding situations where the difference between input power levels is large and the OAs are saturated by high-powered input signal(s). These conditions result in small OA gain which reduces the span between OAs.

The algorithm employs the following OA gain model:

$$
G\left(P_{i n}\right)=M I N\left\{G_{0},\left(P_{M A X}-P_{i n}\right)\right\}
$$

where $P_{i n}$ represents the aggregate power of the input signal, and $G_{0}$ is the small-signal gain in $d B$. Both $P_{M A X}$ and $P_{i n}$ 
are in $\mathrm{dBm}$. Assuming flat gain over all the channels, this gain applies to all input wavelengths.

The network is modeled as a connected undirected graph. Each vertex represents an OXC with Drop-and-Continue $(\mathrm{DaC})$ capability. A subset of the nodes are equipped with power splitters and are called Multicast Capable (MC) nodes while others are referred to as Multicast Incapable (MI). Each splitter has complete splitting capability (i.e., the maximum splitting fanout of the node equals, at least, its out-degree). Also, its splitting ratio is fixed (i.e., the incoming signal power is split equally among the output signals).

Each undirected edge is equivalent to two fibers carrying traffic in opposite directions and all fibers support the same set of wavelengths. Wavelength availability is not constrained and wavelength conversion is not supported. Also, OAs can be placed either on-site or on-link. The on-site placement is sparse and it can be at the node's input (Pre-Amplification) or output (Post-Amplification). Based on the notation introduced in [1], our system model is characterized as $S^{s} F^{c} R^{x}-M^{s}$ where the first term consists of three components that represent the sparse, complete and fixed splitting setting, respectively, while the second term represents the sparse on-site amplification.

The heuristic design is based on the following assumptions:

- We employ the As Late As Possible (ALAP) OA placement policy [7][10]. Yet, any other policy can be used.

- Our study deals with propagation, splitting and tapping losses only. Other power loss sources and impairments due to non-linearities and noise are ignored.

- The value of $P_{S e n}$ is assumed to be high enough to cope with the various types of noises and to guarantee an adequate Bit Error Rate (BER).

\section{The Heuristic Algorithm}

The OAP problem comprises of three main subproblems, namely, Routing (R), Wavelength Assignment (WA), and Power Assignment/Amplifiers Placement (PAAP) subproblems. However, solving one subproblem impacts the solutions of the others. We present a new heuristic for the OAP problem, called the Optical-amplifiers Placement (OP) algorithm. The OP algorithm uses a special set of routing cost functions that are defined in terms of the OAs numbers in order to solve these subproblems separately, but without ignoring their interdependency. It also addresses the impact of the Power Sharing concept [11]; which is a result of sharing the available power by wavelengths at the entry point of the links and OAs. While power sharing may result in connection dropping during network operation [11], its impact during network design phase is translated into a change in the Network Power Status (NPS). NPS defines the network condition in terms of its power values at the beginning of each link, as well as the number, locations and gain values of the OAs. Change in NPS can occur because of any of following behaviors:

1) Power Shortage Behavior, which occurs when the power strength decays below $P_{S e n}$, even when the source node's maximum available power is used. This results in adding more OAs, which increases the network cost.
2) Gain Dropping Behavior, which describes the case when routing more sessions over a link results in dropping the gain of at least one OA which can yield to changing the strengths of its passing signals. As these signals traverse in the network, a sequence of changes in the signals strength and other OAs gains can occur, which might result in violating the power constraints. Consequently, the service of one or more sessions can get disrupted.

3) Power Adjustment behavior, which defines the case when the power values assigned to an already provisioned light-forest(s) is changed in order to maintain the power symmetry and maximum total power constraints.

Due to its dynamic nature, handling NPS is a complicated power management issue, especially with high traffic demand. The OP algorithm tackles this issue by allowing changes to occur to the NPS while efficiently processing their impacts.

The OP algorithm is designed as an iterative algorithm where solutions are optimized at two algorithmic levels. At the lowest level, namely, the light-forest construction level, the sub-forest can expand to new destinations using alternative paths, which allows exploring bigger solution space. Also, the algorithm constructs more than one light-forest per session, and the one with the least cost is then placed in the network.

At the light-forest placement level, two operation modes are adopted, namely, Fixed and Adaptive modes. In the Fixed mode, light-forests are constructed once based on the initial NPS and then they are placed in the network according to their costs. With the Adaptive mode, placing each light-forest in the network is followed by reconstructing the remaining unprovisioned light-forests based on the latest NPS. The rerouting operation allows to account for the impact of lightforests on each other, which improves the solution accuracy.

\section{A. Cost Functions Definitions}

The following cost functions are used by the OP algorithm:

- The current link cost is defined as the current number of OAs needed over the link, including any Post-/PreAmplifiers at the link's source/sink nodes, respectively.

- The current network cost is computed as the total number of OAs over all the links in the network.

- The cost of the path that connects a destination to the subforest is defined as the change in the network cost that results if this path is used for expanding the sub-forest.

- The session cost is also calculated as the change in the network cost if its light-forest is placed in the network.

Using these cost functions for routing creates a connection between the R and PAAP subproblems which reflects positively on the solutions quality. Also, these cost functions reflect the latest changes in the NPS which is essential for the solution accuracy. Moreover, the definitions of the path and session costs is effective in capturing the influence between the various connections as it relates the routing and placement decisions to its future consequences. Finally, the link cost function is a positive increasing function, i.e., the link cost increases as more of its channels become occupied with routed sessions. This is useful in balancing the load in the entire network. 


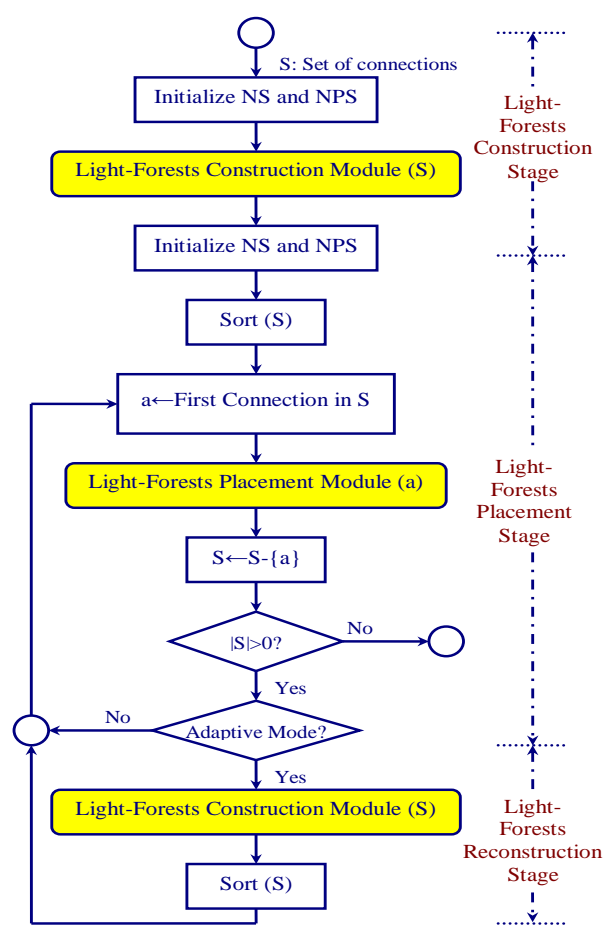

Fig. 1. Basic Operation of the OP Algorithm.

\section{B. OP Algorithm Operation}

The basic operation of the OP algorithm is depicted in Figure 1 and it consists of three main stages, namely, the Light-Forests Construction (LFC), the Light-Forests Placement (LFP) and the Light-Forest Reconstruction (LFR) Stages. The core operation of the LFC and LFR stages is the LightForest Construction Module (LFCM) which is shown in Figure 2 while the core operation of the LFP stage is the Light-Forest Placement Module (LFPM) which is depicted in Figure 3.

The LFC stage starts by initializing the Network Status (NS), NPS, and set $S$. NS determines the channels/links status in the network and set $S$ determines the set of unprovisioned sessions. LFCM is invoked to construct the light-forest for each session in $S$. NS and NPS are then re-initialized and set $S$ is sorted according to its sessions' cost. LFP stage is then invoked for the first session, $a$, in $S$ to place its light-forest in the network. NS, NPS, and $S$ are updated and the algorithm continues with the remaining sessions in $S$ using the Fixed or Adaptive modes. In the Fixed mode, the algorithm places all the initial light-forests constructed in the LFC stage without changing them. However, the Adaptive mode invokes the LFR stage in order to construct new light-forests for all the sessions in $S$ based on the latest network conditions (NS/NPS). After sorting $\mathrm{S}$, algorithm repeats until it is empty.

\section{Light-Forest Construction Module (LFCM)}

LFCM is responsible for constructing one light-forest per multicast session in $S$ based on the recent NS and NPS. Each light-forest is constructed separately as if it is the only light-

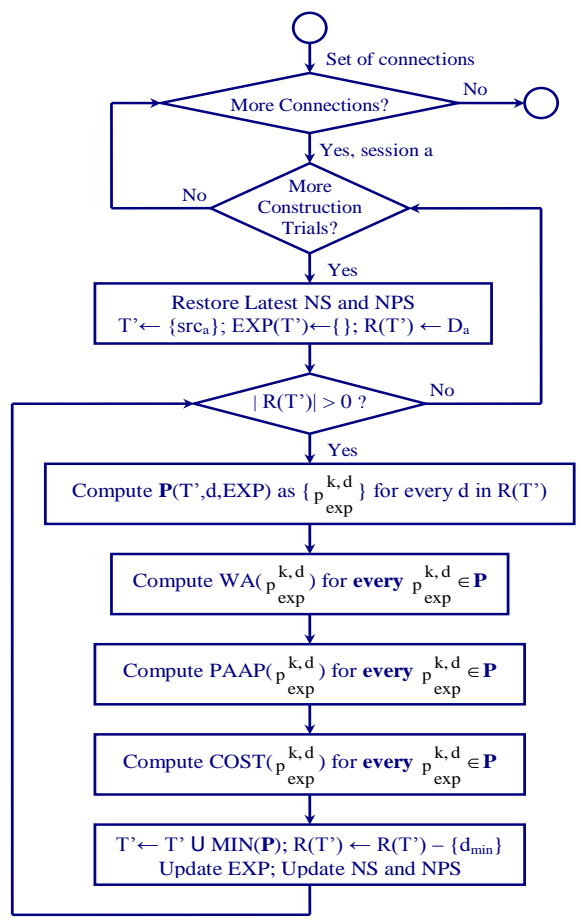

Fig. 2. Light-Forest Construction Module (LFCM)

forest in the system. The module computes multiple lightforests per session and it chooses the one of the least cost.

As shown in Figure 2, each construction trial starts by restoring the latest NS and NPS. Light-forest construction is performed iteratively using an extended version of the Member-Only Heuristic (MOH) [12]. Initially, the light-forest structure, called sub-forest or T', includes the source node only, $\operatorname{src} c_{a}$. With each iteration, T' expands by attaching a new member to $T$ ' via an eligible light-forest node. The set of eligible nodes, called the expandable nodes, or $\operatorname{EXP(T'),~}$ consists of the source node, light-forest MC nodes and leaf nodes. The module stops when all remaining nodes, $\mathrm{R}(\mathrm{T}$ '), are included in the light-forest. During each iteration, the following operations are performed:

\section{1- Path Computation (PC):}

In this step, $k$ alternative paths are computed from each unconnected destination, $d$, to each expandable node in $\operatorname{EXP}\left(\mathrm{T}^{\prime}\right)$.

\section{2- Path Wavelength Assignment (PWA):}

The PWA operation is performed for each path computed in step 1 using the First-Fit scheme.

\section{3- Path Power Assignment/Amplifiers Placement (P- PAAP):}

For each path instance, the P-PAAP operation determines the power values and OAs locations over its links. It relies on using a queue structure, called $Q$, which consists of unique entities of the link instances. Using $Q$ proves to significantly reduce the computation and management overheads in [11] by separating the links identities from their power values.

The P-PAAP operation starts by adding the first link of 


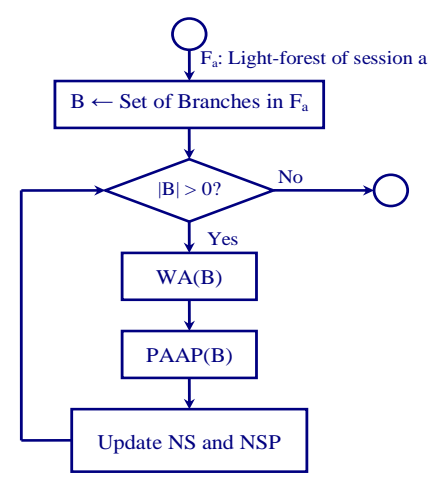

Fig. 3. Light-Forest Placement Module (LFPM)

the path under investigation to $Q$. It then runs iteratively to process the link located at $Q$ 's head, called $l_{q}$. Each iteration starts by ensuring that the power values at the beginning of $l_{q}$ are symmetric and do not violate any power constraint which might require power adjustment. Using these power values, the OAs are then placed over $l_{q}$ based on the ALAP policy. This step might change the previous OAs locations and may add more OAs over $l_{q}$ to accommodate for the change in NPS.

$Q$ is updated with a set of links that belong to the outgoing links of $l_{q}$ 's sink node if they are part of the light-forest of: (1) the current session under investigation, or (2) the other sessions over $l_{q}$ and a new power value is observed at the end of $l_{q}$ that is different than the one from the previous iteration. $l_{q}$ is then removed and the P-PAAP operation continues with the next link in $Q$. It stops when $Q$ becomes empty.

\section{4- Compute Path Cost:}

The cost of all computed paths is computed and T' is expanded using the least cost path.

\section{Light-Forest Placement Module (LFPM)}

The LFPM operation is depicted in Figure 3 and it consists of the WA and PAAP subproblems. These subproblems are similar to the corresponding counterparts introduced in the LFCM, but they are applied at the scale of the light-forest's branches instead of paths. In this context, the WA is performed for each branch of the light-forest using the First Fit policy. Similarly, PAAP operates by start building $Q$ from the first branch's link launched from the source node itself rather than from the first link of the path. Also, LFPM is bypassed in the Adaptive mode, but it must be applied for the Fixed mode.

\section{Numerical Results}

The numerical results presented here are obtained by implementing the $\mathrm{OP}$ algorithm using $\mathrm{C}++$. The experiments are conducted using the 14-nodes NSFNET used in [1] with two splitters at nodes 5 and 8 . The multicast group size follows a uniform distribution between 1 and the number of nodes. Node membership in each session is determined using uniform distribution of all the nodes except the source node. Also, the descending-order policy is used for sorting the set of

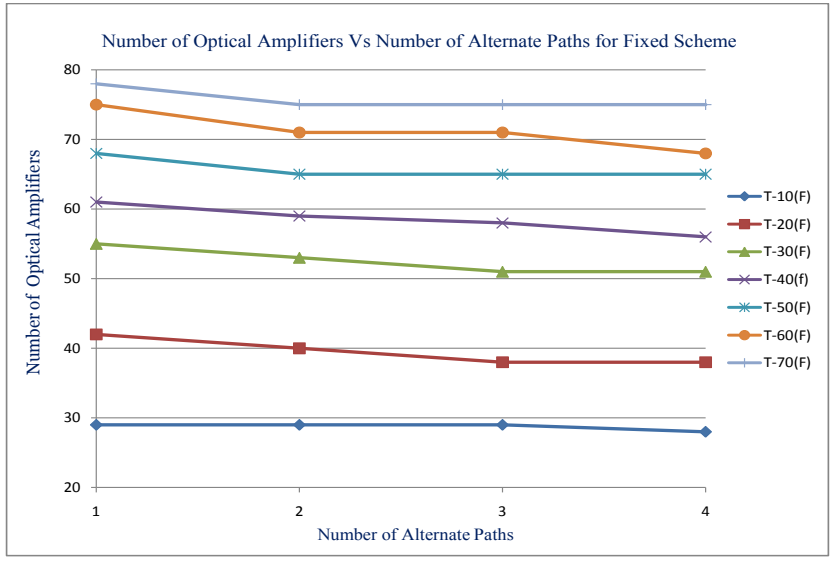

Fig. 4. Impact of Using Alternate Routing on the Number of OAs for the Fixed Scheme at Different Traffic Loads. The notation $T-i(F)$ indicates that traffic load $(T)$ is $i$ sessions for the Fixed $(F)$ scheme.

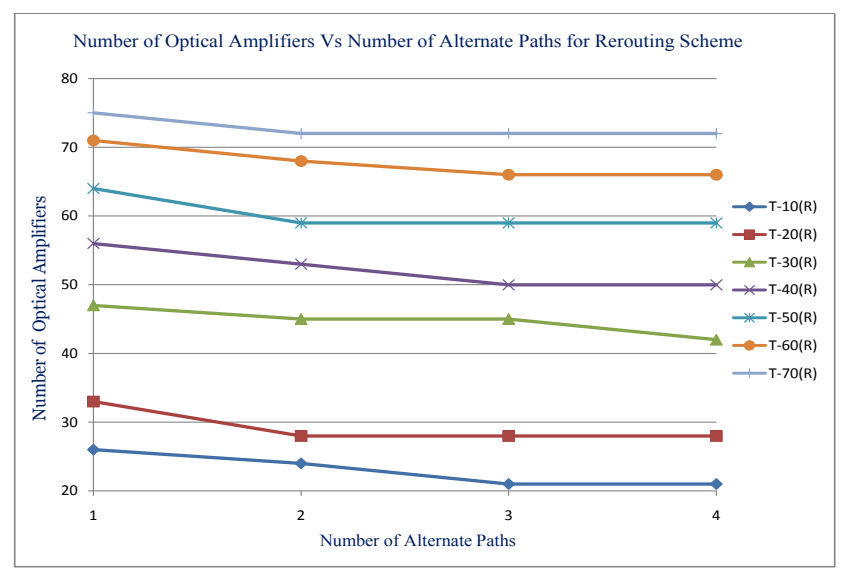

Fig. 5. Impact of Using Alternate Routing on the Number of OAs for the Adaptive Scheme at Different Traffic Loads. The notation $T-i(R)$ indicates that traffic load $(T)$ is $i$ sessions for the Adaptive (or Rerouting, $R$ ) scheme.

connections constructed in the LFC stage. Finally, we run the OP algorithm for at least 10 times for each problem instance and the solution with the minimum number of OAs is chosen.

The solution quality is measured in terms of the number of OAs, $|O A|$, and network resources needed. Two network resources are considered in our study, namely:

1) The maximum number of distinct channels used over any link; this is referred to as $\psi$.

2) The number of links used in constructing all the lightforests; this is referred to as $\mathrm{t}$.

The impact of the OP heuristic on $|O A|$ is depicted in Figures 4 and 5 for the Fixed and Adaptive modes, respectively. $|O A|$ increases as the traffic load increases. However, for each traffic load, $|O A|$ decreases with the increase in the computation complexity. For example, when traffic load is 40 sessions, Figure 4 shows that the solution improved gradually from 61 to 56 OAs as number of alternate paths increased from 1 to 4 . By using the Adaptive scheme only, $|O A|$ drops from 61 to 56, as shown in Figure 5. More improvement could be 


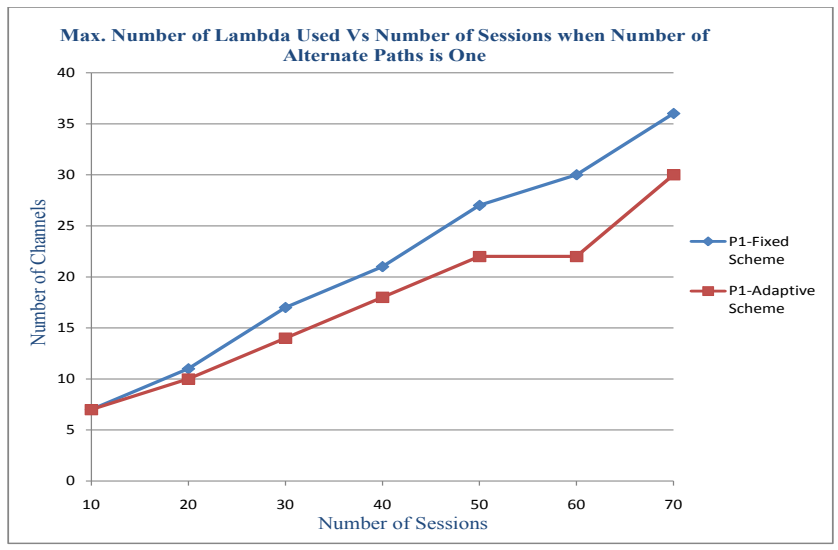

Fig. 6. Maximum number of used channels $(\psi)$ at different traffic loads for the Fixed and Adaptive schemes when number of alternate paths is 1.

achieved when both schemes are employed jointly and $|O A|$ drops to 50 OAs when 4 alternate paths are used.

The contribution of each scheme to the solution quality is measured by the factor $\Delta$ and it is demonstrated in Table I. We define $\Delta$ as the difference in $|O A|$ obtained by subtracting the solution produced when alternate routing scheme with 4 paths is used alone from the solution produced when Adaptive rerouting scheme is used alone. Table I shows that $\Delta$ is a nonnegative number; hence, using the Adaptive scheme alone is as good as or even better than using 4 alternate paths under the Fixed mode. Also, $\Delta$ is highly affected by the traffic load. Results in Table I shows that Adaptive scheme performs best when the traffic load is moderate (i.e., 20-30 sessions).

Figures 6 and 7 depict the network resources consumed by both schemes when the number of alternate paths is one. As shown in Figure 6, the Adaptive scheme uses fewer $\psi$. The saving in $\psi$ increases with the increase in traffic load until it reaches 60 sessions and it then starts to decrease. This behavior can be understood with the aid of Figure 7 which shows that the Adaptive scheme uses less $€$. However, the gap in $€$ decreases when traffic load increases since the Adaptive scheme tends to use more links to accommodate more traffic which directly results in consuming less number of channels per link. This behavior continues until all the available 42 network links are used when traffic load equals 60 sessions. Beyond this point, the Adaptive scheme starts to utilize the available channels and the gap in $\psi$ starts to shrink.

\section{CONCLUSIONS}

We proposed a new heuristic scheme, called the OP algorithm, to solve the OAP problem under AOM. The algorithm

TABLE I

THE RELATIVE PERFORMANCE OF USING ADAPTIVE METHOD ALONE WITH RESPECT TO ALTERNATE ROUTING AT DIFFERENT TRAFFIC LOAD.

\begin{tabular}{|c|c|c|c|c|c|c|c|}
\hline Traffic load (sessions) & 10 & 20 & 30 & 40 & 50 & 60 & 70 \\
\hline$\Delta(O A s)$ & 2 & 5 & 4 & 1 & 0 & 0 & 0 \\
\hline
\end{tabular}

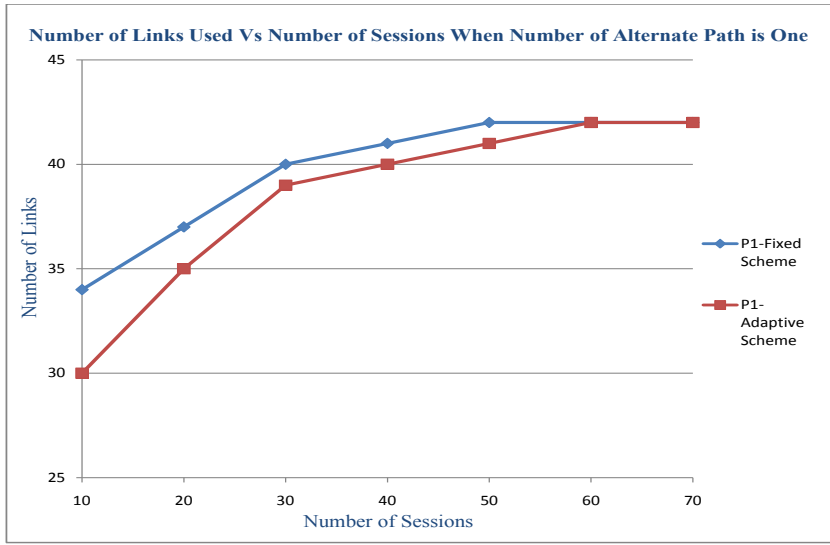

Fig. 7. Number of used links ( $\mathrm{E}$ ) at different traffic loads for the Fixed and Adaptive schemes when number of alternate paths is 1 .

achieves efficient solutions by employing two schemes. In the first scheme, it uses alternate routing for light-forest construction while the second scheme allows light-forest reconstruction. The numerical results show that the network cost decreases with more computation power. Nevertheless, using adaptive scheme alone outperforms the fixed scheme with maximum alternate routes and utilizes less network resources.

\section{REFERENCES}

[1] A. Hamad, T. Wu, A. Kamal, and A. Somani, "Multicasting protocols for wavelength routing networks," Computer Networks, vol. 50, no. 16, pp. 3105-3164, 2006.

[2] L. Sahasrabuddhe and B. Mukherjee, "Light trees: optical multicasting for improved performance in wavelength routed networks," IEEE Commun. Mag., vol. 37, no. 2, pp. 67-73, 1999.

[3] D. Yang and W. Liao, "Design of light-tree based logical topologies for multicast streams in wavelength routed optical networks," in IEEE INFOCOM 03, vol. 1, 2003, pp. 32-41.

[4] M. Dallaali, M. Premaratne, and A. Lowery, "Cost optimal strategies for placement of amplifiers in a point to point optical link," in Internation Conf. on Numerical Simulation of Semiconductor Optoelectronic Devices, 2006, pp. 69-70.

[5] A. Tran, R. Tucker, and N. Boland, "Amplifier placement methods for metropolitan wdm ring networks," in J. Lightwave Technol., vol. 22, no. 11,2004 , pp. $2509-2522$.

[6] A. Hamad and A. Kamal, "Optimal power-aware design of all-optical multicasting in wavelength routed networks," in Proc. IEEE ICC 04, vol. 3, 2004, pp. 1796-1800.

[7] B. Ramamurthy, J. Iness, and B. Mukherjee, "Optimizing amplifier placements in a multiwavelength optical lan/man: the unequally powered wavelengths case," IEEE/ACM Trans. Networking, vol. 6, no. 6, pp. 755767, 1998.

[8] A. Fumagalli, G. Balestra, L. Valcarenghi, M. John, and C. Qiao, "Optimal amplifier placement in multi-wavelength optical networks based on simulated annealing," in Opt. Engr., vol. 3531, pp. 268-279.

[9] A. Sripetch and P. Saengudomlert, "Optimization for optical network designs based on existing power grids," IEICE Transactions on Communications, vol. E91-B, no. 3, pp. 689-699, 2008.

[10] B. Ramamurthy, J. Iness, and B. Mukherjee, "Optimizing amplifier placements in a multiwavelength optical lan/man: the equally poweredwavelengths case," J. Lightwave Technol., vol. 16, no. 9, pp. 1560-1569.

[11] A. Hamad and A. Kamal, "Routing and wavelength assignment with power aware multicasting in wdm networks," in Proc. IEEE Broadnets 05, vol. 1, 2005, pp. 31-40.

[12] X. Zhang, J. Y. Wei, and C. Qiao, "Constrained multicast routing in wdm networks with sparse light splitting," J. Lightwave Technol., vol. 18, no. 12 , pp. $1917-1927,2000$ 